\title{
Measurements of $B$-mode polarization of the cosmic microwave background from 500 square degrees of SPTpol data
}

J. T. Sayre, ${ }^{1,2,3}$ C. L. Reichardt, ${ }^{4}$ J. W. Henning, ${ }^{5,6}$ P. A. R. Ade, ${ }^{7}$ A. J. Anderson, ${ }^{8}$ J. E. Austermann, 9,2 J. S. Avva, ${ }^{10}$ J. A. Beall, ${ }^{9}$ A. N. Bender, ${ }^{5,6}$ B. A. Benson, ${ }^{8,6,11}$ F. Bianchini, ${ }^{4}$ L. E. Bleem, ${ }^{5,6}$ J. E. Carlstrom, ${ }^{6,12,5,11,13}$ C. L. Chang, ${ }^{6,5,11}$ P. Chaubal, ${ }^{4}$ H. C. Chiang, ${ }^{14,15}$ R. Citron, ${ }^{16}$ C. Corbett Moran, ${ }^{17}$ T. M. Crawford ${ }^{6,11}$ A. T. Crites, ${ }^{6,11,18}$ T. de Haan, ${ }^{10,19}$ M. A. Dobbs ${ }^{14,20}$ W. Everett, ${ }^{1}$ J. Gallicchio ${ }^{6,21}$ E. M. George,${ }^{22,10}$ A. Gilbert, ${ }^{14}$ N. Gupta, ${ }^{4}$ N. W. Halverson, ${ }^{1,2}$ N. Harrington, ${ }^{10}$ G. C. Hilton, ${ }^{9}$ G. P. Holder ${ }^{23,24,20}$ W. L. Holzapfel,,${ }^{10}$ J. D. Hrubes, ${ }^{16}$ N. Huang, ${ }^{10}$ J. Hubmayr, ${ }^{9}$ K. D. Irwin, ${ }^{25,26}$ L. Knox, ${ }^{27}$ A. T. Lee, ${ }^{10,19}$ D. Li ${ }^{9,25}$ A. Lowitz, ${ }^{11}$ J. J. McMahon, ${ }^{28}$ S. S. Meyer, ${ }^{6,12,11,13}$ L. M. Mocanu, ${ }^{6,11}$ J. Montgomery, ${ }^{14}$ A. Nadolski, ${ }^{23,24}$ T. Natoli, ${ }^{11,6,29}$ J. P. Nibarger, ${ }^{9}$ G. Noble ${ }^{14}$ V. Novosad, ${ }^{30}$ S. Padin,,${ }^{6,11,18}$ S. Patil, ${ }^{4}$ C. Pryke, ${ }^{31}$ J. E. Ruhl, ${ }^{3}$ B. R. Saliwanchik, ${ }^{3,32}$ K. K. Schaffer,,${ }^{6,13,33}$ C. Sievers, ${ }^{16}$ G. Smecher, ${ }^{14,34}$ A. A. Stark, ${ }^{35}$ C. Tucker, ${ }^{7}$ K. Vanderlinde, ${ }^{29,36}$ T. Veach, ${ }^{37}$ J. D. Vieira, ${ }^{23,24}$ G. Wang, ${ }^{5}$ N. Whitehorn, ${ }^{38}$ W. L. K. Wu, ${ }^{6}$ and V. Yefremenko ${ }^{5}$

(SPTpol Collaboration)

\footnotetext{
${ }^{1}$ Department of Astrophysical and Planetary Sciences, University of Colorado, Boulder, Colorado 80309, USA

${ }^{2}$ Department of Physics, University of Colorado, Boulder, Colorado 80309, USA

${ }^{3}$ Physics Department, Center for Education and Research in Cosmology and Astrophysics, Case Western Reserve University, Cleveland, Ohio 44106, USA

${ }^{4}$ School of Physics, University of Melbourne, Parkville, Victoria 3010, Australia

${ }^{5}$ High Energy Physics Division, Argonne National Laboratory, 9700 S. Cass Avenue, Argonne, Illinois 60439, USA

${ }^{6}$ Kavli Institute for Cosmological Physics, University of Chicago, 5640 South Ellis Avenue, Chicago, Illinois 60637, USA

${ }^{7}$ Cardiff University, Cardiff CF10 3XQ, United Kingdom

${ }^{8}$ Fermi National Accelerator Laboratory, MS209, P.O. Box 500, Batavia, Illinois 60510, USA

${ }^{9}$ NIST Quantum Devices Group, 325 Broadway Mailcode 817.03, Boulder, Colorado 80305, USA

${ }^{10}$ Department of Physics, University of California, Berkeley, California 94720, USA

${ }^{11}$ Department of Astronomy and Astrophysics, University of Chicago, 5640 South Ellis Avenue, Chicago, Illinois 60637, USA

${ }^{12}$ Department of Physics, University of Chicago, 5640 South Ellis Avenue, Chicago, Illinois 60637, USA

${ }^{13}$ Enrico Fermi Institute, University of Chicago, 5640 South Ellis Avenue, Chicago, Illinois 60637, USA

${ }^{14}$ Department of Physics, McGill University, 3600 Rue University, Montreal, Quebec H3A 2T8, Canada

${ }^{15}$ School of Mathematics, Statistics \& Computer Science, University of KwaZulu-Natal, Durban, South Africa

${ }^{16}$ University of Chicago, 5640 South Ellis Avenue, Chicago, Illinois 60637, USA

${ }^{17}$ TAPIR, Walter Burke Institute for Theoretical Physics, California Institute of Technology, $1200 \mathrm{E}$ California Boulevard, Pasadena, California 91125, USA

${ }^{18}$ California Institute of Technology, MS 249-17, 1216 E. California Boulevard, Pasadena, California 91125, USA

${ }^{19}$ Physics Division, Lawrence Berkeley National Laboratory, Berkeley, California 94720, USA

${ }^{20}$ Canadian Institute for Advanced Research, CIFAR Program in Gravity and the Extreme Universe, Toronto, Ontario, M5G 1Z8, Canada

${ }^{21}$ Harvey Mudd College, 301 Platt Blvd., Claremont, California 91711, USA

${ }^{22}$ European Southern Observatory, Karl-Schwarzschild-Str. 2, 85748 Garching bei München, Germany

${ }^{23}$ Astronomy Department, University of Illinois at Urbana-Champaign, 1002 W. Green Street, Urbana, Illinois 61801, USA

${ }^{24}$ Department of Physics, University of Illinois Urbana-Champaign, 1110 W. Green Street, Urbana, Illinois 61801, USA

${ }^{25}$ SLAC National Accelerator Laboratory, 2575 Sand Hill Road, Menlo Park, California 94025, USA

${ }^{26}$ Dept. of Physics, Stanford University, 382 Via Pueblo Mall, Stanford, California 94305, USA

${ }^{27}$ Department of Physics, University of California, One Shields Avenue, Davis, California 95616, USA

${ }^{28}$ Department of Physics, University of Michigan, 450 Church Street, Ann Arbor, Michigan 48109, USA

${ }^{29}$ Dunlap Institute for Astronomy \& Astrophysics, University of Toronto, 50 St George Street, Toronto, Ontario, M5S 3H4, Canada
} 


\author{
${ }^{30}$ Materials Sciences Division, Argonne National Laboratory, \\ 9700 S. Cass Avenue, Argonne, Illinois 60439, USA \\ ${ }^{31}$ School of Physics and Astronomy, University of Minnesota, \\ 116 Church Street S.E. Minneapolis, Minnesota 55455, USA \\ ${ }^{32}$ Department of Physics, Yale University, P.O. Box 208120, New Haven, Connecticut 06520-8120, USA \\ ${ }^{33}$ Liberal Arts Department, School of the Art Institute of Chicago, \\ 112 S Michigan Avenue, Chicago, Illinois 60603, USA \\ ${ }^{34}$ Three-Speed Logic, Inc., Victoria, British Columbia, V8S 3Z5, Canada \\ ${ }^{35}$ Harvard-Smithsonian Center for Astrophysics, 60 Garden Street, \\ Cambridge, Massachusetts 02138, USA \\ ${ }^{36}$ Department of Astronomy \& Astrophysics, University of Toronto, 50 St George Street, \\ Toronto, Ontario, M5S 3H4, Canada \\ ${ }^{37}$ Department of Astronomy, University of Maryland College Park, Maryland 20742, USA \\ ${ }^{38}$ Department of Physics and Astronomy, University of California, Los Angeles, California 90095, USA
}

(Received 23 April 2020; accepted 3 June 2020; published 22 June 2020)

We report a $B$-mode power spectrum measurement from the cosmic microwave background (CMB) polarization anisotropy observations made using the SPTpol instrument on the South Pole Telescope. This work uses $500 \mathrm{deg}^{2}$ of SPTpol data, a five-fold increase over the last SPTpol $B$-mode release. As a result, the bandpower uncertainties have been reduced by more than a factor of two, and the measurement extends to lower multipoles: $52<\ell<2301$. Data from both 95 and $150 \mathrm{GHz}$ are used, allowing for three crossspectra: $95 \mathrm{GHz} \times 95 \mathrm{GHz}, 95 \mathrm{GHz} \times 150 \mathrm{GHz}$, and $150 \mathrm{GHz} \times 150 \mathrm{GHz}$. $B$-mode power is detected at very high significance; we find $P(B B<0)=5.8 \times 10^{-71}$, corresponding to a $18.1 \sigma$ detection of power. With a prior on the galactic dust from Planck, WMAP and BICEP2/Keck observations, the SPTpol $B$-mode data can be used to set an upper limit on the tensor-to-scalar ratio, $r<0.44$ at $95 \%$ confidence (the expected $1 \sigma$ constraint on $r$ given the measurement uncertainties is 0.22 ). We find the measured $B$-mode power is consistent with the Planck best-fit $\Lambda$ CDM model predictions. Scaling the predicted lensing $B$-mode power in this model by a factor $A_{\text {lens }}$, the data prefer $A_{\text {lens }}=1.17 \pm 0.13$. These data are currently the most precise measurements of $B$-mode power at $\ell>320$.

DOI: $10.1103 /$ PhysRevD.101.122003

\section{INTRODUCTION}

Measurements of cosmic microwave background (CMB) anisotropy are a cornerstone of modern cosmology. After recombination at $z \sim 1100$, the overwhelming majority of $\mathrm{CMB}$ photons have freely streamed to observers today. The anisotropy we see primarily arises from fluctuations in the density of the primordial universe during recombination. Thus, measurements of these photons offer us a snapshot of the universe in its infancy.

The CMB is polarized at approximately the $10 \%$ level, due to Thomson scattering off free electrons illuminated by local radiation quadrupoles. At $\ell>10$, polarization is sourced by quadrupole moments that start growing in the primordial plasma and affect the local environment of the photons and electrons as they begin decoupling during recombination. Being driven by scalar (density) perturbations, the resulting full-sky polarization field has even parity analogous to electric fields, following a gradient-like polarization pattern commonly referred to as " $E$-modes." $E$-mode polarization of the CMB has been measured with high precision by, e.g., Henning et al. [[1] hereafter H18], Louis et al. [2] and Planck Collaboration et al. [3], adding information to the temperature spectrum $[3,4]$ both by approximately doubling the number of modes that can be measured on the sky and extending the measurement to smaller angular scales due to the comparatively lower foreground levels in polarization.

In addition to $E$-modes, there are also odd-parity, curllike polarization pattern components, called " $B$-modes." An early prediction of inflation was that there would be a stochastic background of gravitational waves on superhorizon scales [5]. Such gravitational waves would imprint a $B$-mode signature on $\mathrm{CMB}$ polarization peaking at $\ell<100$. The search for the inflationary gravitational wave signal in the polarization of the CMB is a matter of intense current interest, as an unambiguous detection would rule out some alternatives to the inflationary paradigm and yield information on what caused inflation by constraining the shape of the inflaton potential. The best current limit on the inflationary gravitational wave, parametrized as the tensorto-scalar ratio $\mathrm{r}$, is $r<0.06$ at 95\% CL [6], and comes from a combination of data from BICEP2/Keck, Planck, WMAP, and other experiments.

Finally, observers today see a distorted version of the primordial map of the $\mathrm{CMB}$ radiation at recombination due 
to the gravitational lensing of CMB photons by large-scale structure. The small deflections introduced by gravitational lensing do not preserve the even-parity of the initial $E$-mode map, and transform a portion of the $E$-mode power into so-called "lensing $B$-modes." The amplitude of the lensing $B$-mode spectrum depends on the integrated gravitational potential, $\phi$, along the line of sight [7], making it a useful probe of the growth of structure. In particular, the CMB lensing signal can help constrain the sum of the neutrino masses, as larger rest masses increase the expansion rate and thereby suppress growth $[8,9]$.

The first measurement of lensing $B$-modes came from cross-correlating the observed $B$-modes to a template constructed from CMB $E$-modes and a CIB-derived $\phi$ map, as described in Hanson et al. [10]. Since then, direct measurements of the CMB $B$-mode power spectrum have been made by BICEP2/Keck [6,11], SPTpol [12] (hereafter K15), POLARBEAR [13,14] and ACTpol [2,15].

The lensing $B$-mode signal, while cosmologically interesting in its own right, is also a contaminating foreground in any search for inflationary gravitational waves. Improved measurements of the lensing signal also facilitate "delensing" analyses [16], whereby the lensing portion of the $B$-mode signature can be subtracted off, leaving as the residual any potential inflationary gravitational wave $B$-mode signature [e.g., 17,18].

In this work, we present an improved measurement of the $B$-mode power spectrum in the multipole range $52 \leq \ell \leq 2301$ from the SPTpol $500 \mathrm{deg}^{2}$ survey. While the analysis follows the methods in K15 closely, we use five times more sky area in this work (reducing bandpower uncertainties by approximately $\sqrt{5}$ ), and extend the measurement to lower multipoles in order to constrain the inflationary gravitational wave power as well as measuring lensing $B$-modes.

We describe the SPTpol instrument and survey in Sec. II. We discuss the reduction of the time-ordered data in Sec. III, the map-making in Sec. IV, and the power spectrum estimator in Sec. V. We test the data for systematic biases in Sec. VI and then present the resulting bandpowers in Sec. VII. We discuss the implications for cosmology in Sec. VIII, and conclude in Sec. IX.

\section{THE SPTPOL INSTRUMENT AND SURVEY}

The South Pole Telescope (SPT) is a 10-meter diameter, off-axis Gregorian telescope located at the Amundsen-Scott South Pole Station $[19,20]$ that was designed to make highprecision maps of the $\mathrm{CMB}$ with arcminute-scale resolution. The SPTpol instrument replaced the earlier SPT-SZ instrument and was used for observations on the SPT from early 2012 to the end of 2016 (this work uses data through 2015). SPTpol consists of 1536 polarization-sensitive transition edge sensor (TES) bolometers cooled to $250 \mathrm{mK} ; 1176$ with bands centered at $150 \mathrm{GHz}$ and 360 at $95 \mathrm{GHz}$. Pairs of these bolometers that are fed by a common feedhorn form optical pixels, with each bolometer coupled to orthogonal linear polarizations. Full information on the detectors can be found in Henning et al. [21] and Sayre et al. [22].

This work uses SPTpol observations of a $500 \mathrm{deg}^{2}$ field spanning -50 to -65 degrees in declination and $22 \mathrm{~h}$ to $2 \mathrm{~h}$ in right ascension. The $150 \mathrm{GHz}$ data was previously used in the $E$-mode power spectrum measurement by Henning et al. [1], and we refer the reader to that work for a detailed description of the observing strategy. Briefly, we observe the field with a series of back-and-forth raster scans at constant declination, following each raster scan by an approximately 9 arcminute declination step until the full declination range has been covered. Starting declinations are staggered or "dithered" between observations to smooth out the coverage pattern of the field. From April 2013 to May 2014, we observed the field using a "lead-trail" scan strategy which split the field in half in right ascension. From May 2014 onwards, we moved to a full field scan strategy. With a full field scan, we could increase the scan speed (and shift sky signals of interest to higher frequencies) at the cost of losing the ability to difference the two half-maps to remove any ground contamination. Note that we find no indication of significant ground contamination and simply combine the lead and trail maps into complete field observations.

\section{DATA REDUCTION}

The data reduction pipeline used for this work is based on the one used by previous SPTpol power spectrum measurements (K15, Crites et al. [23], hereafter C15, H18). In the following section, we will present a brief overview of the components of the data reduction pipeline, highlighting differences from the procedures used in the works mentioned earlier.

We construct maps using the same procedure as outlined in $\mathrm{C} 15, \mathrm{~K} 15$, and H18. For this work our maps are in the Lambert azimuthal equal-area projection with 1.5 arcminute pixels. Briefly, the reduced, weighted detector timeordered-data are bandpass filtered, combined with pointing information, and binned into the appropriate on-sky map pixel. Binned maps are then cleaned with a second set of cleaning routines before being Fourier transformed and the frequency-domain maps decomposed into the $E$ - and $B$-mode basis. The resulting frequency-domain maps are binned into pseudopower spectra.

\section{A. Calibration of time-ordered data}

The first step is to clean, calibrate and characterize the time-ordered data (TOD). The detector TOD have some response to signals seen by near-by detectors due to electrical "cross-talk." Unlike K15 which dealt with cross-talk at the map level, we begin by removing crosstalk between detectors from each detector's time-ordered 
data. This process is described in H18, and is based on building up a decorrelation matrix from individual detector observations of the Galactic HII region RCW38.

Next, we calibrate the individual detector TOD to CMB brightness temperature in a two-step process. The first step calibrates single detectors using a combination of the response to an internal chopped calibration source and observations of RCW38. This process was first described in Schaffer et al. [24] and used in previous SPT results. We add a second step because we find that calibrated, pixel-differenced timestreams contain excess residual power at low data frequencies, corresponding to large angular scales on the sky. This excess is believed to be due to atmospheric fluctuations. To reduce this residual power, we calculate a small correction to each detector's RCW38-derived calibration by finding the scaling factors between detectors in a pixel pair that minimize the low-frequency noise in their differenced timestreams. For each scan across the field, we calculate the factors $c_{x, y}$ that minimize the pair-differenced noise between 0.1 and $0.3 \mathrm{~Hz}^{1}$ for every pixel. To conserve the mean calibration across the pair, we impose the requirement that $c_{x}+c_{y}=2$ (as $c_{x, y}=1$ represents the initial RCW38 calibration). We then take the average $c_{x, y}$ value for each detector across all scans, considering only values where $0.5<c_{x, y}<1.5$.

It is also crucial to determine the polarization angle of each detector. We use the same measured response angles and polarization efficiencies derived for the $100 \mathrm{~d}$ data in $\mathrm{C} 15$ based on measurements of a polarized source $3 \mathrm{~km}$ away from the telescope. A series of systematic tests, described in both $\mathrm{K} 15$ and $\mathrm{C} 15$, yield an uncertainty on our per-detector angles of $1^{\circ}$, along with a $0.5^{\circ}$ statistical uncertainty from the fits. A correlated error in the detector angles will mix power between $E$ and $B$ modes, and is handled by looking at the $E B$ spectrum. The mean polarization efficiency is $97 \%$ with a statistical uncertainty of $0.7 \%$.

Finally, we calculate the weight that the time-ordered data from each detector in an observation should be given when making maps. These weights are based on each detector's PSD between $1-3 \mathrm{~Hz}^{2}$ We difference left-going and right-going scans before calculating this PSD to null any true sky signal, and average the PSD across all pairs of scans in the observation.

\section{B. Time ordered data filtering}

To reduce the contribution of atmospheric $1 / f$ noise to coadded maps, we filter long-wavelength modes, which are

\footnotetext{
${ }^{1}$ This frequency range corresponds approximately to $\ell=33$ to 200.

${ }^{2}$ The $1-3 \mathrm{~Hz}$ frequency range corresponds roughly to $\ell \in$ $[300,900]$ for a full field observation and $\ell \in[700,2100]$ for a lead-trail observation.
}

expected to be dominated by atmospheric signals in our data, from individual detector time ordered data. We use as our filtering basis functions Legendre polynomials, up to order 5 for lead-trail observations and order 9 for full-field observations, the same values as in H18. Each raster scan across the field is filtered over the same range in RA, and the modes removed correspond to spatial modes on the sky at a multipole of less than approximately $\ell$ of 50 .

\section{Data cuts}

We flag and remove low-quality or pathological data at both the time-ordered data and map levels. For instance, these flags remove data from periods when a detector is not properly biased, or when observing conditions drastically reduce sensitivity to sky signals. These cuts are summarized below.

\section{Time ordered data cuts}

Before binning into maps, we remove data from detectors with corrupted performance as determined by a series of cuts that are very similar to those described in C15 and K15. There are 96 (249) detectors out of a total of 360 (1176) for the $95 \mathrm{GHz}(150 \mathrm{GHz})$ arrays that fail cuts on each of our 4122 observations. For the set of remaining "live" detectors, we cut those with anomalous performance according to a series of metrics measured from TOD. The metrics are listed below in the order they are applied to each observation, so a cut detector is counted only by the first test it fails. The average percentage of detectors at $95 \mathrm{GHz}$ $(150 \mathrm{GHz})$ removed are noted after each cut.

(1) Timestream errors, like a failure to properly bias the detector TES into its transition, readout electronics failure affecting the detector channel, and unphysical calibration of the detector time ordered data into $K_{\mathrm{CMB}}$ units; 7.6 (5.8).

(2) Anomalously low or unphysically high response to either the chopped internal calibration source or a dip in telescope elevation (which modulates atmospheric loading); 2.1 (0.28).

(3) TOD weights, thresholds are empirically set based on distributions of weight values for all observations, which include variability of sky and telescope conditions, to remove unphysical values; 4.3 (3.1).

(4) Low-frequency noise, measured between 0.0 and $0.4 \mathrm{~Hz}$ in individual detector TOD, calibrated in $K_{\mathrm{CMB}}$ units. Detectors more than $4 \sigma$ away from the mean for all detectors in a given observation are removed; 3.2 (1.6).

(5) Broadband noise, measured from the mean power spectral density of all scans for each detector, integrated between $0.4 \mathrm{~Hz}$ and $3 \mathrm{~Hz}$, with detectors more than $5 \sigma$ away from the central value being cut; $0.6(0.8)$.

(6) Full pixel, which removes every bolometer whose pixel partner was cut. This cut ensures that 
polarization maps are not corrupted by an uneven sampling of the $Q$ and $U$ maps; 3.6 (1.7).

In addition to the above cuts of a detector's data for a full observation, we flag individual raster scans where a detector experiences a "glitch," defined as an anomalous difference $(>5 \sigma)$ between two subsequent data samples. The distribution of sample-to-sample differences is calculated for the entire focal plane for each scan, and any detector with a difference more than five standard deviations away from the mean has its data for that specific scan cut.

\section{Cuts on low-frequency map noise}

While we weight the data from individual detectors according to their noise PSDs (see Sec. III A), this will not necessarily account for observations with unusual levels of correlated noise between detectors, for instance due to atmospheric fluctuations. We therefore also implement a cut based on the low-frequency noise in each observation's map. We calculate the angular power spectrum of each map's Stokes $T, Q$, and $U$ components, constructing a metric, $\Xi_{\alpha}$, defined by:

$$
\begin{aligned}
C_{\ell<300}^{\alpha, i} & =\sum_{\ell<300} C_{\ell}^{\alpha, i} \\
\Xi_{\alpha} & =\frac{C_{\ell<300}^{\alpha, i}}{\operatorname{median}\left(C_{\ell<300}^{\alpha, i}\right)}
\end{aligned}
$$

where $C_{\ell}^{\alpha, i}$ is the angular power spectrum of map $i$ and $\alpha=(\mathrm{TT}, \mathrm{QQ}$, or $\mathrm{UU})$. We cut any map where the lowfrequency polarization noise is ten times higher than the median noise, i.e., if either $\Xi_{Q Q}$ or $\Xi_{U U}$ is greater than 10 .

After removing maps with anomalously high low-frequency noise, we are left with 3628 good individual half- or full field observations from a total of 4341 performed between March 2013 and November 2015. Because cuts are applied independently to the lead and trail maps from 2013 , sometimes only one of a lead-trail pair passes. We combine these orphan half-observations with the nearestin-time counterpart, and cut the eight half-field maps where a counterpart cannot be found. We are left with 3620 maps, which, when the lead-trail pairs are combined into observations of the full field, yields a total of 2890 complete observations of the field: 730 in the lead-trail format, 2160 in the full-field format.

\section{Beams}

We measure the instrumental angular response function ("beam") with observations of Venus made in January 2013, that are convolved by an estimate of the effect of pointing uncertainties in the CMB fields. A two-dimensional Gaussian is fit to each $1^{\circ}$-by- $1^{\circ}$ Venus map made with third-order polynomial subtraction, and they are coadded with their best-fit peak pixels aligned. The resulting two-dimensional map is then convolved with a two-dimensional Gaussian with widths that are determined from fits to a series of bright point sources in the CMB field, measured with nominal pointing information. This second step accounts for the "jitter" associated with our nominal pointing model. Thus the convolved Venus map includes the effects of the true instrumental angular response and the variations in pointing over the course of our observations.

The small size of the Venus maps and the use of polynomial filtering of the time-ordered-data mean that the measured beam only has high-fidelity information above $\ell \approx 500$. However, as described in H18, we find that the Venus beam profile at large angular scales is in good agreement with an estimate derived using a separate method of cross-spectral analysis between Planck maps and SPTpol maps. As a result, we use the Venus profiles over the full range of multipoles in this analysis. We take the variance among our 8 (13) clean Venus maps at $95 \mathrm{GHz}$ $(150 \mathrm{GHz})$ as our beam errors, with the variance among the 8 cross-maps that include our $95 \mathrm{GHz}$ maps as the error on our $95 \mathrm{GHz} \times 150 \mathrm{GHz}$ beam. We marginalize over seven beam parameters in the fits, representing the seven largest eigenvectors of the beam covariance matrix. We find our results are robust to doubling the assumed beam uncertainty in Section VIII B.

\section{MAP PROCESSING}

We apply further processing at the map level before calculating the power spectrum. In particular, we filter out the monopole temperature leakage and signals fixed in RA. For computational and coverage reasons, we also bundle together many observations of the field into a bundle map. We apodize these maps and mask bright radio sources. We then convert the $Q / U$ maps to $E / B$ modes using the $\chi_{B}$ estimator from Smith and Zaldarriaga [25].

\section{A. Map bundles}

In order to smooth coverage and reduce the computational demands for later processing steps, we combine the maps into a series of 50 "bundles." For each observation format (lead-trail and full), we combine all constituent maps into a single one and measure its total map weight. We then divide that total map weight by 50 to get the target per-bundle summed weight. We then order the maps chronologically (by the start time of the lead observation for mismatched lead-trail pairs) and combine them sequentially until each bundle is as close as possible to the target per-bundle weight. The lead-trail-only and full-only bundles are used in the systematics tests described in Sec. VI A. For the final data products, we combine the first lead-trail bundle with the first full bundle and so on until we have 50 bundles, each composed of a lead-trail and full bundle, for both the $95 \mathrm{GHz}$ and $150 \mathrm{GHz}$ data. 


\section{B. Apodization and point source masking}

We apodize the maps before Fourier transforming in order to reduce mode-coupling due to a sharp edge and to downweight the low-weight and high-noise pixels at the edge of the map. For simplicity, we use the same apodization mask for all map bundles. Thus we begin by finding the intersection across all bundles ${ }^{3}$ of the set of pixels with a weight at least $30 \%$ of the median weight. The combined coverage mask is then reduced by a 4 arcminute border at its edges to reduce edge effects before apodizing the result with a 90 arcminute wide cosine taper. The resulting effective sky area after application of the apodization mask is 458.3 square degrees.

While we will marginalize over an unknown Poisson point source power in the parameter fitting, we choose to mask the brightest sources (with intensity fluxes $>50 \mathrm{mJy}$ at $150 \mathrm{GHz}$ ) to minimize the shot noise.

\section{Map-space processing}

At this point, we have $T, Q$, and $U$ maps which we want to transform into a $B$-mode map. Some systematic sources of apparent $B$-modes are most readily dealt with in the map domain. Thus we project out a temperature map template from each $Q / U$ map and remove a template based on the azimuthal signal before converting from $Q / U$ to $E / B$ maps.

\section{Monopole temperature leakage deprojection}

Miscalibrating the gains of two detectors in a pixel causes a scaled copy of the temperature map to leak into the $Q$ and $U$ polarization maps. This "monopole" leakage is straightforward to measure and remove in the $Q, U$ maps before they are transformed to $E$ and $B$. To estimate the leakage, we first construct two half-depth coadds by adding up all even-numbered bundles and all odd-numbered bundles. The resulting maps are crossed to produce TQ and TU pseudo-cross-spectra, which are each normalized by the TT pseudo-cross-spectrum. Normalized cross-spectral ratios are then averaged over a chosen ell range, $\ell=$ $100-3000$ in this work, yielding coefficients, $\hat{Q}$ and $\hat{U}$, of the T-to-P monopole leakage. The coefficients, $\hat{Q}=0.0263$ (0.0162), $\hat{U}=-0.0215$ (0.0095) for 95 (150) GHz maps, are insensitive to the exact choice of ell range, but we choose the range where our expected cosmological signal is maximal. The uncertainty on these factors is 0.0010 . Each bundle $Q$ and $U$ map then has the appropriately scaled version of its own $T$ map subtracted from it.

\footnotetext{
${ }^{3}$ We actually use a larger bundle set, resulting in a smaller intersection region, to ensure that the mask is also appropriate for all null tests. Namely, we include the individual bundles in the lead-trail vs full and left-going vs right-going splits.
}

\section{RA template removal}

We find evidence for scan-synchronous signals in our bundle maps, with an rms of approximately 4 (1.5) $\mu K$ in 95 (150) $\mathrm{GHz} Q$ and $U$ maps, similar in scale to the signals in the 100d field, described in K15. To remove it, we measure a one-dimensional profile by binning bundle map pixels by their RA location and smoothing the result by a 1-degree wide Hann window. The resulting profile is then re-projected into two dimensions along the elevation direction and subtracted from the bundle $Q$ and $U$ maps.

\section{D. $E$ and $B$ mode maps}

After applying the real-space processing steps described above to our $T, Q$, and $U$ maps, we decompose them into harmonic space $T, E$, and $B$ maps for further processing and power spectrum estimation. We construct the $E$-mode maps with the standard transformation [26],

$$
E_{\ell}=Q_{\ell} \cos \left(2 \phi_{\ell}\right)+U_{\ell} \sin \left(2 \phi_{\ell}\right)
$$

where $Q_{\ell}$ and $U_{\ell}$ are Fourier transforms of the processed and apodized real-space $Q$ and $U$ maps, and $\phi_{\ell}=$ $\arctan \left(\ell_{x} / \ell_{y}\right)$. A generic effect of the $E-B$ decomposition with partial sky coverage is the presence of ambiguous modes, which mix $E$-mode sky signal into the constructed $B$-mode map. To minimize this effect, we use the $\chi_{B}$ estimator from Smith and Zaldarriaga [25]. Our final Fourier-space $B$-mode maps are thus constructed according to:

$$
B_{\ell}=\frac{\mathcal{F}\left(W\left(\left(\partial_{X}^{2}-\partial_{Y}^{2}\right) Q+2 \partial_{X Y}^{2} U\right)\right)}{\sqrt{\alpha_{\ell}}}
$$

where $\mathcal{F}$ represents the Fourier transform, $\alpha_{\ell}=l(l-1) \times$ $(l+1)(l+2), \mathrm{W}$ is the apodization mask and the derivatives of $Q$ and $U$ are the intermediate $\chi_{B}$ maps. The derivatives are calculated using finite differences with a $5 \times 5$ pixel kernel centered on each map pixel. The resulting $B$-mode maps are shown in Fig. 1.

\section{POWER SPECTRUM ESTIMATION}

We estimate the $B$-mode bandpowers using a pseudo- $\mathrm{C}_{\ell}$ cross-spectrum method [see K15, 27,28]. Starting from the cleaned Fourier-space $B$-mode maps of the last section, we average across the set of all cross-spectra to measure the pseudospectrum. We then correct this pseudospectrum for effects such as the finite sky coverage to create an unbiased estimate of the true $B$-mode power on the sky.

The binned pseudo- $\mathrm{C}_{\ell}$ spectrum is calculated from the mean of the cross-spectra between all bundle map pairings:

$\hat{D}_{b}^{x \times y}=\frac{1}{N_{x \times y}} \sum_{i \neq j} \sum_{\ell \in b}\left(\frac{\ell(\ell+1)}{2 \pi} \operatorname{Re}\left(W_{\ell}\left(B_{\ell}^{x, i} B_{\ell}^{y, j *}\right)\right)\right)$. 

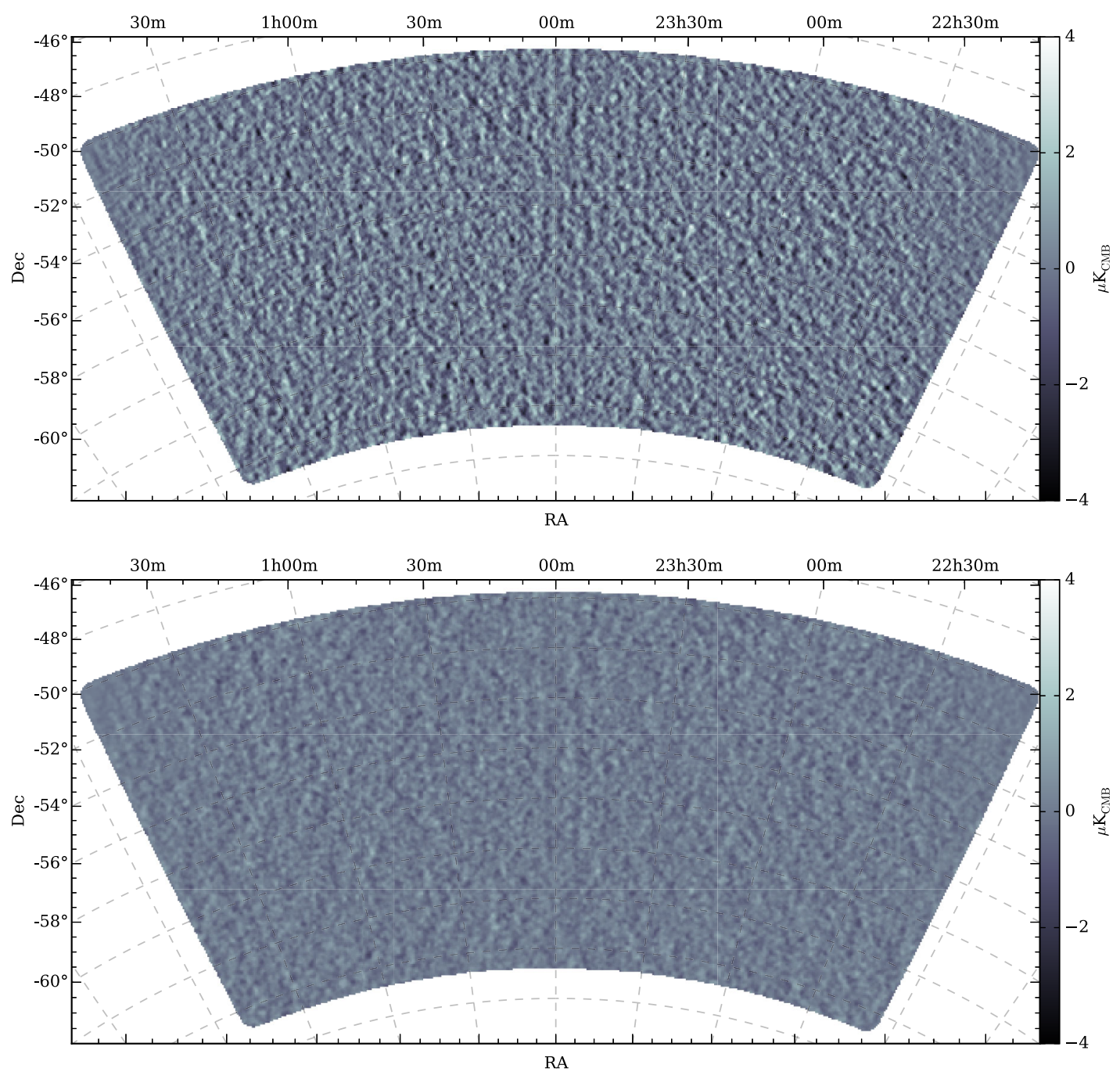

FIG. 1. The $B$-mode sky maps used in the work, shown as transformed back from frequency-domain maps with all processing steps applied. The top panel is $95 \mathrm{GHz}$ while the bottom panel is $150 \mathrm{GHz}$. Both maps are noise-dominated on all angular scales.

Here $x$ and $y$ denote 95 or $150 \mathrm{GHz}$, and $i$ or $j$ denote the bundle number. The Fourier-space $B$-mode map for frequency $x$ and bundle $i$ is $B_{\ell}^{x, i}$, while $W_{\ell}$ is a Wiener-filterderived mode weighting. $N_{x \times y}$ is the number of crossspectra: there are a total of 1225 cross spectra for the 90 and $150 \mathrm{GHz}$ auto-spectra and 2450 for the $95 \mathrm{GHz} \times$ $150 \mathrm{GHz}$ spectrum.

This binned pseudo-spectrum $\hat{D_{b}}$ is related to the true binned spectrum $D_{b}$ by:

$$
\hat{D_{b}}=K_{b b^{\prime}} D_{b^{\prime}}+A_{b}+A_{T B}+A_{E B} .
$$

We refer to these binned spectra as bandpowers. Here $A_{b}$ captures additive biases to the $B$-mode power created for instance by the map filtering, while $A_{T B}$ is to allow for effects such as very low amplitude polarised beam sidelobes that are not in the simulations. In principle, $A_{b}$ should be written as a function of $\left\{C_{\ell}^{T T}, C_{\ell}^{E E}, C_{\ell}^{T E}\right\}$ but the temperature and $E$-mode power spectra have already been measured to high precision so we fix $A_{b}$ to the expectation for the fiducial cosmology. We remove $A_{T B}$ and $A_{E B}$ by subtracting

$$
D_{b^{\prime}}^{B B}=D_{b^{\prime}}^{\prime B B}-\frac{\left(D_{b^{\prime}}^{T B}\right)^{2}}{D_{b^{\prime}}^{T T}}-\frac{\left(D_{b^{\prime}}^{E B}\right)^{2}}{D_{b^{\prime}}^{E E}}
$$

In principle, the $A_{E B}$ term could be introduced by an miscalibration of the polarization angles, as discussed in Sec. III A. In practice, it is very close to zero suggesting that the fiducial polarization angles are accurate. The maximum value of this term for a $150 \mathrm{GHz}$ bandpower is $0.001 \mu \mathrm{K}^{2}$. Note that we handle each spectrum (e.g., TT, BB) independently for both simulations and real data, and do not include off-diagonal blocks such as $(\mathrm{TT}, \mathrm{BB})$ in the modecoupling matrices. The kernel matrix $K_{b b^{\prime}}$ encapsulates the effects of mode-mixing due to partial sky coverage, and the 
suppression of power by the instrumental beam and map filtering.

\section{A. Estimating the additive biases}

We measure the induced additive bias in the $B$-mode power spectrum by measuring the observed $B$-mode power in a suite of $100 T E$-only simulations (see Sec. VE). The additive bias can be understood by considering the ambiguous modes that are created by the interaction of the partial sky coverage and edge apodization with the polynomial filtering applied to the TOD. These ambiguous modes mix the $E$-mode power into $B$-modes, particularly at low angular multipoles. Because the $E$-mode power spectrum is tightly constrained and we can accurately simulate the TOD processing, we can determine the expectation value for the additive bias, $A_{b}$, using the $200 T E$ simulations. As with the real data, we first subtract the $T-B$ leakage estimate [Eq. (6)] from each individual $T E$ simulation. In the lowest multipole bin, the additive bias is larger than the expected $B$-mode power, quickly falling and becoming negligible at higher angular multipoles. Specifically, the additive bias is $\sim 35 \%$ of the expected power in second bin $(\ell \in[152,301]), \sim 10 \%$ in the third bin, and about $3 \%$ at higher multipoles. We also subtract the estimated $A_{b}$ from the TEB simulations used for estimating the bandpower covariance, with the variations about the mean additive bias adding to the sample variance estimate.

\section{B. Estimating the kernel matrix}

We also need to calculate the kernel matrix $K_{b b^{\prime}}$ in order to apply its inverse and recover an unbiased estimate of the true sky power spectrum. The kernel matrix, which includes the effects of binning, TOD filtering and mapmaking, instrumental beams, and mode mixing due to edge apodization and finite sky coverage, can be written as:

$$
K_{b b^{\prime}}=P_{b \ell^{\prime}}\left(M_{\ell \ell^{\prime}} T_{\ell^{\prime}} \mathcal{B}_{\ell^{\prime}}^{2}\right) Q_{\ell^{\prime} b^{\prime}} .
$$

Here $P_{b \ell}$ and $Q_{\ell b}$ are the binning and unbinning operators [27] that translate between bandpower-space and native $\ell$ space, and $M_{\ell \ell}$ is the mode-mixing matrix that describes the $\ell$-space mixing induced by finite sky coverage and edge apodization of our field. In this work, given the relative lack of features in the $B$-mode power spectrum at the current signal-to-noise, we make the simplifying approximation that the mode-mixing matrix is diagonal. The measurement of the azimuthally-averaged beam $\mathcal{B}_{\ell}$ was described in Sec. IIIC3, and $T_{\ell}$ is the filter transfer function described next.

\section{Filter transfer function}

We estimate the effect of the TOD filtering and the mapmaking process using a set of noiseless simulated $\mathrm{CMB}$ skies. Each sky realization is passed through the full TOD processing, map-making and conversion from $Q / U$ to Wiener-filtered $E B$ maps. The $B B$ power spectrum is then calculated for each of the $100 T E$ and $100 T E B$ skies (see Sec. VE). The $T E$ skies are required to estimate any additive bias to the $B$-mode spectrum. As with the real data, we subtract the $T-B$ leakage estimate [Eq. (6)] from each individual $T E$ and $T E B$ simulation. We subtract the mean $T E B$-mode spectrum from the mean TEB $B$-mode spectrum. As we have assumed a diagonal mode-mixing matrix, we simply take the ratio of this cleaned spectrum to the product of the beam function $\mathcal{B}_{\ell}^{2}$ and combined CMB and foreground spectrum $C_{\ell}$ as the one-dimensional transfer function $T_{\ell}$.

\section{Bandpower covariance}

The bandpower covariance includes contributions from sample and noise variance. The noise variance is estimated through the covariance between individual cross-spectrum realizations from our ensemble of noise-only bundles, while the sample variance is calculated from the scatter in the set of autospectra of the simulated signal-only $T E B$ map realizations described in Sec. VE. Note that these simulations are for $r=0$. We combine these two estimates together according to:

$$
\begin{aligned}
& \Xi_{b b}^{X X}=2 \gamma_{b}\left(S_{b}^{X X}+N_{b}^{X X}\right)^{2} \\
& \Xi_{b b}^{X Y}=\gamma_{b}\left[\left(S_{b}^{X Y}\right)^{2}+\left(S_{b}^{X X}+N_{b}^{X X}\right)\left(S_{b}^{Y Y}+N_{b}^{Y Y}\right)\right],
\end{aligned}
$$

where $b$ denotes the multipole bin, $X / Y$ represent either 95 or $150 \mathrm{GHz}$, and $S$ and $N$ are the signal and noise power respectively. The prefactor, $\gamma_{b}$, accounts for the number of modes in each multipole bin. For the noise terms (both $N^{2}$ and $S N$ ), we make the simplifying assumption that the bandpower covariance matrix between two different multipole bins $b \neq b^{\prime}$ is zero, i.e., the matrix is block diagonal. While we expect a small degree of correlation between neighboring bins due to the finite sky coverage, this correlation is minimized because the chosen bin sizes are significantly wider than the expected angular multipole resolution for the field size. Thus the noise variance should be approximately diagonal. As a check on this assumption, we compute the $\chi^{2}$ statistic of our bandpowers relative to a fiducial cosmology spectrum with both the block-diagonal covariance and the version preserving the noisy estimates of the off-diagonal structure. We find a $\Delta \chi^{2}$ of approximately 1 for the 21 bandpowers. We allow the sample variance terms $\left(S^{2}\right)$ to have an arbitrary shape since the lensing-induced $B$-modes should have correlations between multipoles. We also stress that bin-bin correlations are included for the beam and calibration uncertainties, which are dealt with separately.

We add to the covariance an estimate of the $1 \sigma$ variations in the T-to-P monopole leakage terms in Sec. IV C 1. 


\section{Power spectrum calibration}

As described in Sec. III A, we initially calibrate our detector data in units of $\mathrm{CMB}$ brightness temperature by fitting to a known-brightness source. We further refine our calibration by cross-correlating SPTpol $T$ - and $E$-mode maps to the published Planck maps over our nominal observation region, as described in H18. Because we use nearly identical data sets and processing options as in $\mathrm{H} 18$, we take the median of that work's calibration posterior as our $150 \mathrm{GHz}$ polarization calibration factor, $P_{\text {cal }}^{150}$. The uncertainty on $P_{\text {cal }}^{150}$ is $0.5 \%$ based on the H18 posterior.

As $\mathrm{H} 18$ only used $150 \mathrm{GHz}$ data, we must also extend the known $150 \mathrm{GHz}$ polarization calibration to $95 \mathrm{GHz}$. We do this by constructing an ensemble of ratio spectra between the $95 \times 150 \mathrm{GHz}$ and $150 \times 150 \mathrm{GHz}$ pseudocross spectra:

$$
\epsilon_{\ell, i}=\frac{C_{\ell E E, i}^{95 \times 150}}{C_{\ell E E, i}^{150 \times 150}} \frac{\mathcal{B}_{\ell}^{150}}{\mathcal{B}_{\ell}^{95}}
$$

Here $i$ denotes which cross-spectrum. We average each ratio spectrum over the $\ell$-bins with inverse variance weighting to yield an ensemble of ratio factors, $\epsilon_{i}$, and take as our $95 \mathrm{GHz}$ polarization calibration scaling the value $P_{\text {cal }}^{95}=\left\langle P_{\text {cal }}^{150}\right\rangle\left\langle\epsilon_{i}\right\rangle$. We estimate the uncertainty in $\left\langle\epsilon_{i}\right\rangle$ from the spread in the $\epsilon_{i}$ ensemble, and estimate a combined uncertainty of $5.2 \%$ in the $95 \mathrm{GHz}$ calibration by adding the two uncertainty terms in quadrature. We have also confirmed that the measured E-mode power spectra from these maps at 95 and $150 \mathrm{GHz}$ are consistent with the reported bandpowers in H18. We marginalize over two calibration parameters in all fits, representing the 95 and $150 \mathrm{GHz}$ calibration factors, with priors set from the above calculation.

\section{E. Simulations}

A crucial element of our power spectrum analysis is the use of simulated skies. We start with 100 Lenspix realizations of lensed $T, E, B$ skies, generated from the Planck + WP + high L cosmology in Planck Collaboration et al. [29], and add in Gaussian foregrounds realizations. The foreground terms include polarized Galactic dust with a polarized power of $0.0236 \mu \mathrm{K}^{2}\left(\frac{\ell}{80}\right)^{-0.42}$ and $150 \mathrm{GHz}$; unpolarized thermal Sunyaev-Zel'dovich effect power with an amplitude of $5 \mu \mathrm{K}^{2}$ at $\ell=3000$ and $150 \mathrm{GHz}$; Poisson dusty star-forming galaxies at a power level of $9 \mu \mathrm{K}^{2}$ at $\ell=3000$ and $150 \mathrm{GHz}$ and polarization fraction of 0.025 ; Poisson radio galaxies at a power level of $10 \mu \mathrm{K}^{2}$ at $\ell=$ 3000 and $150 \mathrm{GHz}$ and polarization fraction of 0.025 ; and lastly clustered dusty star-forming galaxies with a power level of $5 \mu \mathrm{K}^{2}$ at $\ell=3000$ and $150 \mathrm{GHz}$. The $\mathrm{CMB}$ and foreground $a_{\ell m}$ 's are combined and convolved with a temporary detector beam profile. The beam used in simulations is an approximation of the production beam described in Sec. IIIC 3, as the beam analysis was not complete when simulations were generated. ${ }^{4}$ At this stage, we make a copy of each set of beam-convolved $a_{\ell m}$ 's and zero its $B$-modes, allowing us to track the leakage of power from $(T, E)$ into $B$ due to our map processing steps. The $T$, $E$, and $B a_{\ell m}$ 's are then converted back into $T, Q$, and $U$ according to our field definition and projected onto a grid of cylindrical coordinates at twice the resolution of our final maps. We then mock-observe the $T, Q$, and $U$ skies to produce pairs of noiseless $T E B$ sky and associated $T E$-only sky maps. The $T$ maps are identical between the two sets, while the $Q$ and $U$ maps differ only by the lack of source $B$ modes in the latter set.

\section{SYSTEMATIC TESTS}

We now turn our attention to potential sources of systematic error in the reported bandpowers. First, we look at a suite of null tests to validate the bandpowers against unexpected systematics. Then, we examine the sensitivity of the power spectrum to possible systematics which were not tested by the null test suite. We find the BB bandpowers are not significantly impacted by systematic biases.

\section{A. Null tests}

To check for systematic contamination in our data, we create difference or null maps that will null the true sky signal while maximizing the potential systematic signal for various potential systematics. For each potential systematic, we start from a set of 100 maps, order the maps according to the relevant statistic, and then difference the first 50 from the second 50 maps to create 50 null maps. We calculate the bandpowers for this 50 null maps, which should be consistent with zero in the absence of systematics.

(1) Azimuth: The CMB field rotates relative to the ground throughout the course of observations, so by bundling maps according to the azimuth angle of the ground under the field during observations, we can isolate contamination from sources at the South Pole station.

(2) Lead-Trail/Full: In addition to changing the raster pattern, which in turn affects the weights of full-field maps, the switch from lead-trail to full observations included increasing the scan speed. Each jackknife bundle consists of a lead-trail bundle differenced

\footnotetext{
${ }^{4}$ The differences between the final and simulation beams are small, typically at the subpercent level with a maximum fractional difference of a few percent.
} 
TABLE I. Null test PTEs.

\begin{tabular}{ll}
\hline \hline Test Statistic & PTE \\
\hline $\max _{f s j}\left(\left|\Sigma_{b} \chi_{b}^{f s j}\right|\right)$ & 0.24 \\
$\max _{f s j}\left(\left(\chi_{b}^{f s j}\right)^{2}\right)$ & 0.60 \\
$\max _{f s j}\left(\Sigma_{b}\left(\chi_{b}^{f s j}\right)^{2}\right)$ & 0.92 \\
$\Sigma_{b f s j}\left(\chi_{b}^{f s j}\right)^{2}$ & 0.13 \\
Global & 0.38 \\
\hline \hline
\end{tabular}

The PTEs listed indicate how often statistic in question is higher in the simulated $\chi$ bandpowers than in our ensemble of jackknife $\chi$ bandpowers. The global PTE indicates how often all four statistics in from a realized ensemble of $\chi$ bandpowers exceed the values from our jackknife bandpowers. The top row $\left.\operatorname{(max}_{f s j}\left(\left|\Sigma_{b} \chi_{b}^{f s j}\right|\right)\right)$ tests for spectra that are preferentially positive or negative. The second row $\left(\max _{f s j}\left(\left(\chi_{b}^{f s j}\right)^{2}\right)\right)$ tests for individual outliers. The third row $\left(\max _{f s j}\left(\Sigma_{b}\left(\chi_{b}^{f s j}\right)^{2}\right)\right)$ is sensitive to null spectra with a larger than expected number of outliers. The fourth row is the total $\chi^{2}$ for all spectra, frequency combinations and null tests. The last row, "Global," is the fraction of simulations that have larger PTEs for all four tests simultaneously.

with a combined bundle of full observations taken at a similar time in subsequent years.

(3) Moon: Jackknife maps are constructed by combining bundles, without respect to lead-trail or full observation strategy, according to the nearness of the moon to the observation field.
(4) Sun: Jackknife maps are constructed similarly to the moon jackknife maps, except using the presence of the sun in the sky, which occurs at both the very beginning and end of each observing season.

(5) Left—Right: Each of the Lead-Trail/Full bundles is constructed from maps consisting of rightgoing-only and leftgoing-only scans, with the two sets of scans usually combined to get a full coverage observations. For this jackknife test, the rightgoing-only and leftgoing-only bundles are differenced, to test for scan-synchronous contamination that depends on the direction of telescope motion. In particular, rightgoing scans always follow an elevation step, so any "wobble" in the telescope due to the elevation motion would stand out in a right-left difference.

We calculate the probability-to-exceed (PTE) of the $\chi^{2}$ values of each set of jackknife bandpowers for each frequency combination $(95 \times 95,150 \times 150$, and $95 \times 150)$ and each sky combination $(B \times B, E \times B$, and $T \times B$ ) relative to a null spectrum. The individual jackknife test PTEs support the case of no contamination, with only two of the 45 PTEs outside the interval of $(0.05,0.95)$. As a further distillation of the $\chi^{2}$ information, the PTEs relative to null of the combined bandpowers for all tests and frequency combinations are $0.67(B \times B), 0.20(E \times B)$, and $0.19(T \times B)$, and 0.38 for all bandpowers across all spectra and frequency combinations.

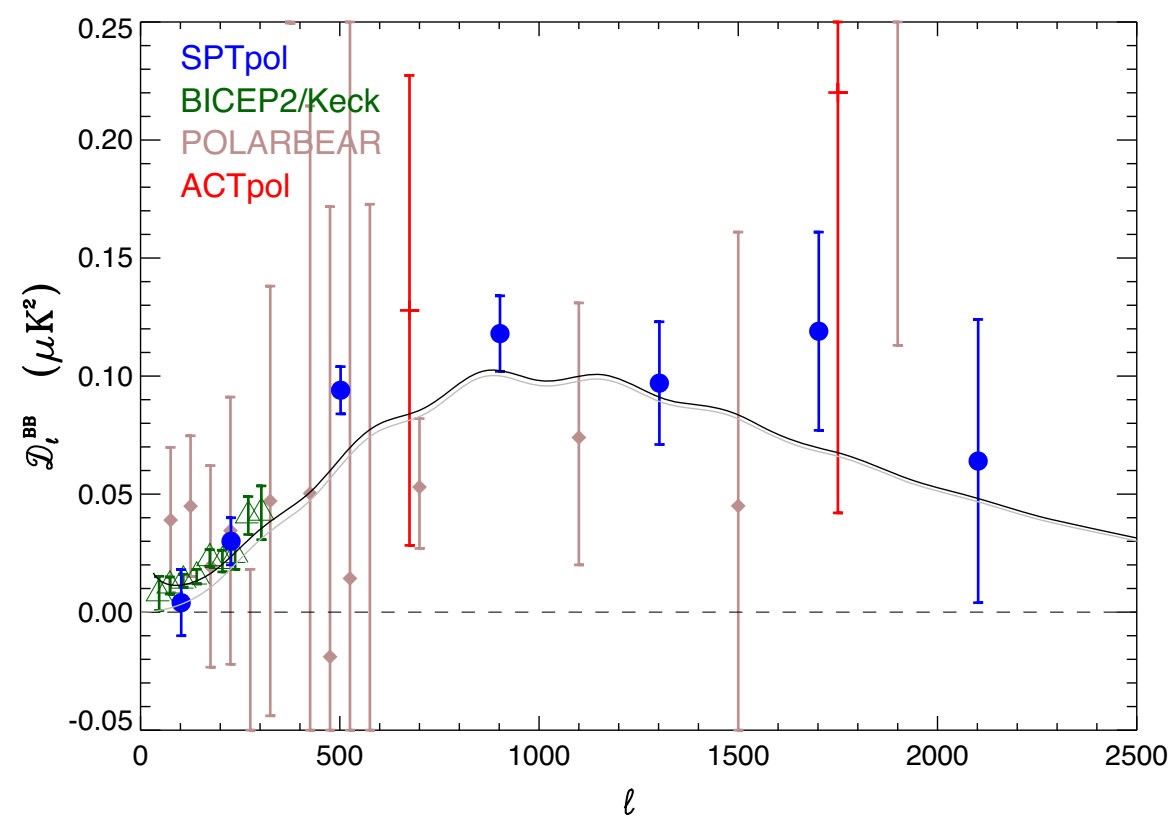

FIG. 2. The $B$-mode bandpowers from the minimum variance combination of the $95 \times 95,95 \times 150$, and $150 \times 150 \mathrm{GHz}$ bandpowers in this work (blue circles) along with the $B$-mode measurements from other experiments. The $150 \mathrm{GHz}$ results for BICEP2/Keck [6] are shown by the green triangles. ACTpol results at $150 \mathrm{GHz}$ [2] are marked by the red crosses, and POLARBEAR measurements at $150 \mathrm{GHz}[13,14]$ by the rosy brown diamonds. The grey line shows the prediction for lensed $B$-mode power for the PLANCK best-fit model, while the black line adds on the best-fit Galactic dust power from BICEP2 Collaboration et al. [6]. The $B$-mode bandpowers from this work are the most precise measurements at $\ell>320$. 
TABLE II. BB bandpowers, $D_{\ell}\left[\mu \mathrm{K}^{2}\right]$.

\begin{tabular}{|c|c|c|c|c|c|c|c|c|c|}
\hline \multirow[b]{2}{*}{$\ell_{\text {center }}$} & \multirow[b]{2}{*}{$\ell$ range } & \multicolumn{2}{|c|}{$95 \times 95$} & \multicolumn{2}{|c|}{$95 \times 150$} & \multicolumn{2}{|c|}{$150 \times 150$} & \multicolumn{2}{|c|}{ Combined } \\
\hline & & $D_{\ell}$ & $\sigma\left(D_{\ell}\right)$ & $D_{\ell}$ & $\sigma\left(D_{\ell}\right)$ & $D_{\ell}$ & $\sigma\left(D_{\ell}\right)$ & $D_{\ell}$ & $\sigma\left(D_{\ell}\right)$ \\
\hline 102 & $52-151$ & 0.043 & 0.123 & 0.018 & 0.032 & -0.000 & 0.015 & 0.004 & 0.014 \\
\hline 227 & $152-301$ & 0.158 & 0.064 & 0.026 & 0.019 & 0.027 & 0.011 & 0.030 & 0.010 \\
\hline 502 & $302-701$ & 0.206 & 0.053 & 0.062 & 0.018 & 0.103 & 0.013 & 0.094 & 0.010 \\
\hline 902 & 702-1101 & 0.313 & 0.081 & 0.106 & 0.029 & 0.111 & 0.020 & 0.118 & 0.016 \\
\hline 1302 & 1102-1501 & 0.369 & 0.125 & 0.019 & 0.045 & 0.119 & 0.032 & 0.097 & 0.026 \\
\hline 1702 & $1502-1901$ & 0.162 & 0.217 & 0.249 & 0.075 & 0.054 & 0.051 & 0.119 & 0.042 \\
\hline 2102 & 1902-2301 & 0.430 & 0.345 & 0.284 & 0.112 & -0.045 & 0.073 & 0.064 & 0.060 \\
\hline
\end{tabular}

The BB bandpowers.

In addition to the basic $\chi^{2}$ PTE tests, we repeat the process described in K15, whereby individual " $\chi$ bandpowers," defined as

$$
\chi_{b}^{f s j} \equiv \frac{C_{b}^{f s j}}{\sigma\left(C_{b}^{f s j}\right)}
$$

are compared to 100000 simulated ensembles generated from unit-width, zero-mean Gaussian distributions. The superscripts represent the spectrum $(f \in\{B B, E B, T B\})$, frequency combination $(s \in\{95 \times 95,95 \times 150,150 \times 150\})$, and null test $(j)$, while the subscript $b$ represents a specific $\ell$ bin. We construct a series of test statistics that probe various potential signatures of systematic contamination, summarized in Table I, and measure how often the statistic as calculated from the simulated $\chi$ bandpowers exceeds the value from a particular jackknife.

We take the values in Table I, summarized by the global $\chi^{2}$ PTE as strong evidence that our data is not contaminated by any of the potentials sources of systematic contamination we investigated.

\section{B. Other possible systematics}

Now we turn to two systematics that are not tested by the jackknives. In contrast to K15, we remove crosstalk directly from time ordered data before binning into maps. We also explicitly remove monopole $T \rightarrow Q U$ leakage from maps before transforming into harmonic space. Thus, the dominant sources of leakage are expected to be $E \rightarrow B$ leakage from filtering. These leakage terms are accounted for in Sec. VA using the observed $B$-modes after filtering the $T E$ only sims, with variance in the leaked power showing up as additional sample variance.

Variations in detector responsivity as a function of the observing elevation would not be detected by jackknife tests. To probe this we generate half-map masks, starting from the nonapodized mask described in Sec. IV B and zeroing either the portion greater or less than declination of $-57.5^{\circ}$. The resulting masks are then apodized with the same parameters as the real data mask and used to estimate two sets of power spectra. We find no evidence of inconsistency between either set of half-map spectra and the full-map spectra, with a $\chi^{2}$ PTE of 0.21 when comparing the subfield bandpowers and their diagonal covariances to the full-field bandpowers.

\section{BANDPOWERS}

The final minimum-variance combination of the debiased bandpowers is compared to the results from other experiments in Fig. 2. The bandpowers for each frequency combination are provided in Table II and plotted in Fig. 4. Above $\ell=300$, the bandpower definitions are identical to $\mathrm{K} 15$, and the two sets of spectra are consistent, with the uncertainties in this work reflecting the expected $\sqrt{5}$ reduction in total variance from greater sky coverage. Along with the bandpowers, both figures show the same Planck best-fit cosmology described in Sec. VIII B.

\section{INTERPRETATION}

We now look at the consistency of the bandpowers with the $\Lambda$ CDM model. While the bandpowers in this work are the best measurements of the $B$-mode power spectrum above $\ell>320$, we do not expect them to substantially restrict the allowed parameter space within the $\Lambda \mathrm{CDM}$ framework. However, these data are interesting as an independent consistency test of the $\Lambda \mathrm{CDM}$ framework and in the implications for inflationary gravitational waves.

\section{A. Parameter fitting}

We use the Markov Chain Monte Carlo (MCMC) package созмомс [30] to fit the bandpowers to a simple model of the form:

$$
D_{\ell}^{\nu_{1} \times \nu_{2}}=r D_{\ell}^{\mathrm{tens}}(r=1)+A_{\mathrm{lens}} D_{\ell}^{\mathrm{lens}}+D_{\ell}^{\mathrm{fg} ; \nu_{1} \times \nu_{2}}
$$

We calculate the two templates $D_{\ell}^{\text {tens }}$ and $D_{\ell}^{\text {lens }}$ using CAMB [31] at a Planck best-fit cosmology: $\left\{\Omega_{b} h^{2}=0.022294\right.$, $\Omega_{c} h^{2}=0.11837, \theta=1.041042, \tau=0.0677, \log A=3.0659$, $\left.n_{s}=0.969\right\}$, with $\sum m_{\nu}=60 \mathrm{meV}$. Details on how to 
install and use the SPTpol likelihood and dataset are available on the SPT website. ${ }^{5}$

The foreground terms included are Galactic dust emission at large angular scales and Poisson power due to polarized emission from extragalactic galaxies at small scales. We have clear predictions for the Galactic dust emission from Planck and BICEP2/Keck; there are only upper limits on the Poisson power as of yet. The functional form of these foreground terms $D_{\ell}^{f g ; \nu_{1} \times \nu_{2}}$ for the $\nu_{1} \times \nu_{2}$ cross-spectra is

$$
\begin{aligned}
D_{\ell}^{f g ; \nu_{1} \times \nu_{2}}= & A_{\ell=80 ; 150 \mathrm{GHz}}^{\text {dust }} f_{\nu_{1}, \nu_{2}}\left(\frac{\ell}{80}\right)^{-0.58} \\
& +A_{\ell=3000 ; \nu_{1} \times \nu_{2}}^{\text {Pois }}\left(\frac{\ell}{3000}\right)^{2}
\end{aligned}
$$

Here the top line has the expression for Galactic dust and the bottom line the expression for the extragalactic power. The frequency dependence of the Galactic dust is encoded in $f_{\nu_{1}, \nu_{2}}$ which we assume to be a grey-body spectrum with temperature $T=19.6 \mathrm{~K}$ and $\beta=1.59$ [32]. Motivated by the recent measurements by BICEP $2 /$ Keck on the same region of sky [6], we place a Gaussian prior on $A_{\ell=80 ; 150 \mathrm{GHz}}^{\text {dust }}=0.0094 \pm 0.0021 \mu \mathrm{K}^{2}$. We also take the angular shape (i.e., $D_{\ell} \propto \ell^{-0.58}$ ) from the best-fit in that work. With only one bin across the relevant angular scales, we do not independently constrain the angular shape of the Milky Way's emission. BICEP2 Collaboration et al. [6] also show that galactic synchrotron is negligible on this field at 95 or $150 \mathrm{GHz}$ for the current uncertainties. We make no assumptions about the spectral dependence of the extragalactic Poisson power and thus have three parameters $A_{\ell=3000 ; \nu_{1}, \nu_{2}}^{\text {Pois }}$ describing the Poisson power at $\ell=3000$ in the $\nu_{1} \times \nu_{2}$ bandpowers. We note that of these foreground terms, the data only shows a significant preference for the $95 \mathrm{GHz}$ Poisson power $\left(\Delta \chi^{2}=11.4\right.$ for 1 d.o.f.); the others are included to estimate realistic uncertainties.

\section{B. Results}

The SPTpol bandpowers are visually consistent with the Planck best-fit $\Lambda$ CDM cosmology with $r=0$. Assuming $r=0$, we find $A_{\text {lens }}=1.17 \pm 0.13$ which is consistent with the expected value of unity. Allowing $r$ to vary as well does not significantly change the $A_{\text {lens }}$ constraints. The $95 \% \mathrm{CL}$ upper limit on $r$ from the SPTpol BB bandpowers only is $r<0.44$. The likelihood curves for $A_{\text {lens }}$ and $r$ are shown in the left and right panels of Fig. 3 respectively. We also calculate the goodness of fit of the model with $r=0^{6}$ to the data; the PTE is low at $2 \%$. This is driven by the three $95 \times 150 \mathrm{GHz}$ bandpowers above $\ell=1102$.

\footnotetext{
${ }^{5}$ http://pole.uchicago.edu/public/data/sayre19/.

${ }^{6}$ Allowing $r$ to vary does not improve the fit quality significantly.
}

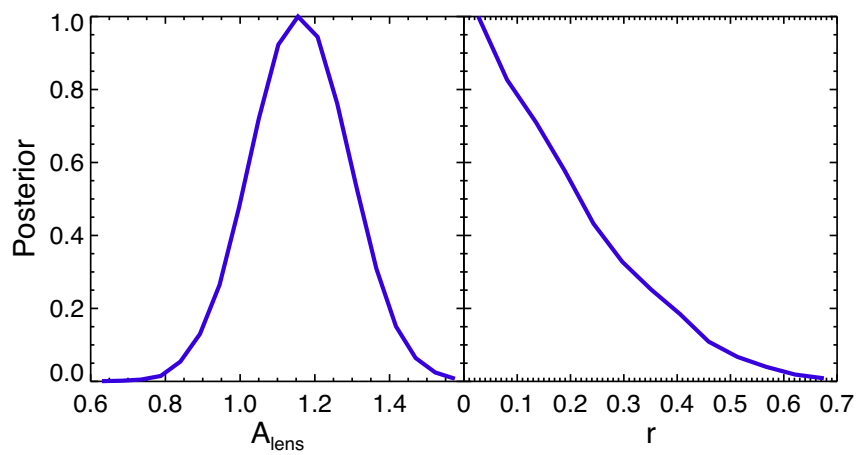

FIG. 3. The measured $B$-mode power spectrum is consistent with the Planck best-fit $\Lambda$ CDM model. On the left, we show the posterior probability for $A_{\text {lens }}\left(A_{\text {lens }}\right.$ rescales the predicted $B$ modes due to lensing), finding it consistent with unity. In the right panel, we show that the posterior probability for the tensor-toscalar ratio $r$ peaks at zero.

Both of these results are robust against the assumed Galactic dust prior. Increasing (or decreasing) the central value of the prior on the Galactic dust power by $50 \%$ only slightly decreases (increases) the central value to $A_{\text {lens }}=$ $1.14 \pm 0.13(1.18 \pm 0.13)$. The resulting $r$ limits go to $r<0.39$ and $r<0.46$ respectively. Removing the external dust prior altogether minimally changes the result to $r<0.43$. Thus the results do not depend closely on the details of the dust prior.

The results are driven primarily by the $150 \times 150 \mathrm{GHz}$ bandpowers, and the $95 \times 95 \mathrm{GHz}$ bandpowers have little weight in the parameter fits. We have confirmed this by removing each frequency combination, $95 \times 95 \mathrm{GHz}$, $95 \times 150 \mathrm{GHz}$, or $150 \times 150 \mathrm{GHz}$, and rerunning the MCMCs. Without the $95 \times 95 \mathrm{GHz}$ bandpowers (which appear high), the recovered 95\% CL limit on $r$ is $r<0.44$. The recovered value is $A_{\text {lens }}=1.13 \pm 0.13$ in this case; the slightly lower median value of $A_{\text {lens }}$ explains the equivalent limit on $r$ with less data. Conversely, dropping the $150 \mathrm{GHz}$ autospectrum nearly doubles the uncertainty on $A_{\text {lens }}$ to 0.25 , and triples the limit on $r$ to $r<1.40$.

It is noticeable in Fig. 4 that the $95 \mathrm{GHz}$ autospectrum appears consistently high, and in turn, as noted in Sec. VIII A, the $95 \mathrm{GHz}$ Poisson power is the only clearly preferred foreground parameter. The default foreground model in this work independently floats the Poisson power in each frequency band, however a natural question is what spectral index is implied by the relative Poisson powers seen between the three bands. To answer this question, we tie together the Poisson terms, assuming that the source fluxes scale as a power law $\nu^{\alpha}$. Note that $\alpha$ is for the source fluxes, not the power. We set a uniform prior of $\alpha \in[-4,0]$. In this model, the upper limit on the Poisson power at $150 \mathrm{GHz}$ actually tightens by about $40 \%$ (and zero is now excluded since the $95 \mathrm{GHz}$ result requires some power). The constraint on alpha is very weak with a $90 \%$ CL range $\alpha \in[-3.2,-0.6]$; the posterior peaks at $\alpha \sim-2.65$. 


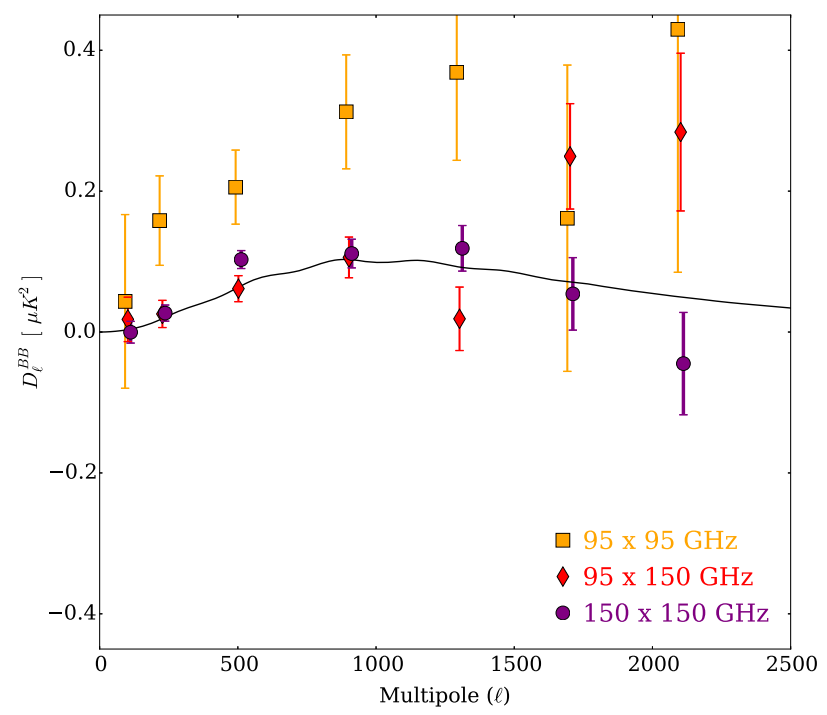

FIG. 4. The $B B$ power spectrum bandpowers from the individual $95 \times 95 \mathrm{GHz}$ (orange squares), $95 \times 150 \mathrm{GHz}$ (red diamonds), and $150 \times 150 \mathrm{GHz}$ (purple circles) spectra. For reference, the expected lensed $B B$ spectrum from the PLANCK +LENSING+WP+HIGHL best-fit model from [33], also used as the source spectrum for simulated skies, is shown as the black solid line.

A spectral index in this range is on the low end of what has been observed for the temperature-selected sources in Mocanu et al. [34], but a large number of synchrotron sources in that work did have $\alpha<-0.6$. We also note that masking sources selected only at $150 \mathrm{GHz}$ (as in this work) would tend to drive this spectral index more negative. A alternative explanation is that the mean squared polarization fraction, $\left\langle p^{2}\right\rangle$, has increased going from 150 to $95 \mathrm{GHz}$. The ratio of the median mean-squared polarization fraction at these two bands from Gupta et al. [35] is 1.3; if one shifts these by one sigma in either direction, the ratio increases to 1.7. Such a shift in $\left\langle p^{2}\right\rangle$ would have a similar effect on the 95 to $150 \mathrm{GHz}$ power as changing the spectral index by 0.6 . It will be interesting to see if this frequency trend in the Poisson $B$-mode power holds up with future measurements, or if the excess power turns out to be the result of a systematic bias in the $95 \mathrm{GHz}$ bandpowers.

We also try fitting the data with doubled calibration and beam uncertainties. As one would expect given the relative size of the bandpower error bars to the beam and calibration uncertainties, this has no impact on the recovered $r$ or $A_{\text {lens }}$ values.

Finally, we quantify the detection significance for cosmological BB power by looking at the probability for negative values of $A_{\text {lens }}$ in a MCMC run at high temperature in order to better sample the extreme tails of the posterior distribution. For the normal case, we find $P\left(A_{\text {lens }}<0\right)=$ $1.8 \times 10^{-18}$, corresponding to a $8.7 \sigma$ detection of positive $A_{\text {lens }}$. In the absence of sample variance, this probability becomes $P\left(A_{\text {lens }}<0\right)=2.6 \times 10^{-29}$, corresponding to a $11.2 \sigma$ detection of lensing $B$-modes. Lastly, to evaluate the detection significance of any $B$-mode power on the sky, we drop sample variance and set the foreground terms to zero. We find $P\left(A_{\text {lens }}<0\right)=5.8 \times 10^{-71}$, corresponding to a $18.1 \sigma$ detection of any $B$-mode power.

\section{Constraints on primordial magnetic fields}

Measurements of the $B$-mode power spectrum also test models that predict primordial magnetic fields (PMFs) or cosmic birefringence [e.g., 36]. Both effects have the observational effect of rotating $E$-modes into $B$-modes, thereby adding $B$-mode power compared to the standard $\Lambda \mathrm{CDM}$ cosmology. Since they are observationally indistinguishable in the bandpowers, we will only quote limits on the PMF power here. These limits can be translated to apply to parity violating processes as well.

We follow the approach of Sutton et al. [37], drawing upon the vector and tensor PMF templates for the CMB power spectra from that work. We add these templates to the calculated CAMB spectra. We assume the initial PMF anisotropy is Gaussian distributed with a nearly scaleinvariant $\left(n_{B}=-2.9\right)$ power law spectrum. Thus there are two free parameters: an overall power normalization $A_{P M F} \propto B_{1 M p c}^{4}$, and a timing parameter for when the PMF is generated $\beta=\ln \left(a_{\nu} / a_{\mathrm{PMF}}\right)$. Here, $B_{1 M p c}^{4}$ is the RMS strength of the PMF over $1 \mathrm{Mpc}$ scales, and $a_{\nu}$ and $a_{\mathrm{PMF}}$ are the scale factors at neutrino decoupling and PMF generation respectively. The chosen template is for $B_{1 M p c}=2.5 \mathrm{nG}$. The timing of PMF generation relative to neutrino decoupling impacts the magnitude of the tensor PMF modes. We follow earlier works [37-39] and set a prior that $\log _{10}\left(a_{\nu} / a_{\mathrm{PMF}}\right) \in[11.513,41.447]$. We find the improved bandpower measurements in this work lead to a 95\% CL upper limit of $A_{P M F}<0.37$. If we instead use the prior range of $\log _{10}\left(a_{\nu} / a_{\mathrm{PMF}}\right) \in[0,16.937]$ from Ade et al. [40], the 95\% CL upper limit becomes $A_{P M F}<0.42$. As the bandpowers have scattered high (i.e., $A_{\text {lens }}=1.17 \pm$ 0.13 in Sec. VIII B), this upper limit from the BB bandpowers of this work alone is equivalent to the limit of $A_{P M F}<0.36$ for $A_{\text {lens }}=1$ that was found by Sutton et al. [37] for the combination of Planck, POLARBEAR, BICEP2/Keck Array and the earlier $100 \mathrm{deg}^{2}$ SPTpol data releases.

\section{CONCLUSIONS}

We present a measurement of the angular power spectrum of CMB $B$-mode polarization from the $500 \mathrm{deg}^{2}$ SPTpol survey. Using three seasons of data, we report 21 bandpowers in seven multipole bins spanning $52 \leq \ell \leq$ 2302 and three frequency combinations: $95 \mathrm{GHz} \times$ $95 \mathrm{GHz}, 95 \mathrm{GHz} \times 150 \mathrm{GHz}$, and $150 \mathrm{GHz} \times 150 \mathrm{GHz}$. These bandpowers represent the most precise direct measurement of $B$-mode power to date at small angular scales 
$(\ell>320)$, and range from angular scales where inflationary gravitational waves may be found to scales dominated by lensing $B$-modes.

We have performed a strict set of null tests to probe the data for unknown systematic errors, and find no evidence for systematic contamination. Astrophysical foreground $B$-modes are a potential concern which we address by marginalizing over a Galactic template (important at low $\ell$ ) and independent Poisson power terms for each frequency band representing polarized extragalactic sources. The Galactic template and prior is based on the measurements of Galactic polarized dust emission reported in BICEP2 Collaboration et al. [6]. The data do not require these foreground terms, except in showing a $3 \sigma$ preference for Poisson power at $95 \mathrm{GHz}$.

Having found no evidence for systematic effects, we quantify the detection significance for astrophysical or cosmological $B$-mode power, and find the data rule out no $B$-mode power at $18.1 \sigma$. Marginalizing over astrophysical foregrounds but still neglecting sample variance, $\mathrm{CMB}$ $B$-mode power, consistent with the expectations for gravitational lensing, is detected at $11.2 \sigma$. We check the data for consistency with the predicted lensing $B$-modes in the Planck best-fit $\Lambda$ CDM model by fitting for an unknown rescaling $A_{\text {lens }}$ of the predicted lensing power. We find $A_{\text {lens }}=1.17 \pm 0.13$, consistent with the expected value of unity in $\Lambda$ CDM.

With bandpowers extending down to $\ell=52$, this work is the first direct search for inflationary gravitational wave $B$-modes with the South Pole Telescope. The bandpowers presented here lead to a 95\% CL upper limit on the tensorto-scalar ratio of $r<0.44$. This limit is close to what should be expected given the experimental sensitivity-we calculate the expected $\sigma(r)$ for these measurement uncertainties is 0.22 . This limit is largely set by the $150 \times$ $150 \mathrm{GHz}$ bandpowers due to the higher map noise level at $95 \mathrm{GHz}$. We can expect further improvements from the new SPT-3G survey on the South Pole Telescope, a $1500 \mathrm{deg}^{2}$ survey that began in 2018 and is expected to reach map depths a factor of several deeper than the data used here [41].

\section{ACKNOWLEDGMENTS}

The South Pole Telescope program is supported by the National Science Foundation through Grant No. PLR1248097. Partial support is also provided by the NSF Physics Frontier Center Grant No. PHY-0114422 to the Kavli Institute of Cosmological Physics at the University of Chicago, the Kavli Foundation, and the Gordon and Betty Moore Foundation through Grant No. GBMF\#947 to the University of Chicago. This work is also supported by the U.S. Department of Energy. The Melbourne authors acknowledge support from an Australian Research Council Future Fellowship (No. FT150100074). J. W. H. is supported by the National Science Foundation under Award No. AST-1402161. W. L. K.W is supported in part by the Kavli Institute for Cosmological Physics at the University of Chicago through Grant No. NSF PHY1125897 and an endowment from the Kavli Foundation and its founder Fred Kavli. B. B. is supported by the Fermi Research Alliance LLC under Contract No. De-AC0207CH11359 with the U.S. Department of Energy. The Cardiff authors acknowledge support from the UK Science and Technologies Facilities Council (STFC). The CU Boulder group acknowledges support from NSF Grant No. AST-0956135. The McGill authors acknowledge funding from the Natural Sciences and Engineering Research Council of Canada, Canadian Institute for Advanced Research, and the Fonds de Recherche du QuébecNature et technologies. The UCLA authors acknowledge support from NSF Grants No. AST-1716965 and No. CSSI1835865. Work at Argonne National Lab is supported by UChicago Argonne LLC,Operator of Argonne National Laboratory (Argonne). Argonne, a U.S. Department of Energy Office of Science Laboratory, is operated under Contract No. DE-AC02-06CH11357. We also acknowledge support from the Argonne Center for Nanoscale Materials. This research used resources of the National Energy Research Scientific Computing Center (NERSC), a U.S. Department of Energy Office of Science User Facility operated under Contract No. DE-AC02-05CH11231. The data analysis pipeline also uses the scientific python stack [42-44] and the HDF5 file format [45].
[1] J. W. Henning, J. T. Sayre, C. L. Reichardt et al., Astrophys. J. 852, 97 (2018).

[2] T. Louis, E. Grace, M. Hasselfield et al., J. Cosmol. Astropart. Phys. 06 (2017) 031.

[3] N. Aghanim, Y. Akrami et al. (Planck Collaboration), arXiv: 1907.12875.

[4] K. Story, E. Leitch, P. Ade et al., Proc. SPIE Int. Soc. Opt. Eng. 8451, 84510T (2012).
[5] A. H. Guth, Phys. Rev. D 23, 347 (1981).

[6] P. A. R. Ade et al. (BICEP2 and Keck Array Collaborations), Phys. Rev. Lett. 121, 221301 (2018).

[7] U. Seljak and M. Zaldarriaga, Phys. Rev. Lett. 82, 2636 (1999).

[8] K. N. Abazajian, K. Arnold, J. Austermann et al., Astropart. Phys. 63, 66 (2015).

[9] Z. Pan and L. Knox, Mon. Not. R. Astron. Soc. 454, 3200 (2015). 
[10] D. Hanson, S. Hoover, A. Crites et al., Phys. Rev. Lett. 111, 141301 (2013).

[11] P. A. R. Ade, Z. Ahmed et al. (BICEP2 and Keck Array Collaborations), Astrophys. J. 811, 126 (2015).

[12] R. Keisler, S. Hoover, N. Harrington et al., Astrophys. J. 807, 151 (2015).

[13] S. Adachi, M. A. O. A. Faúndez, K. Arnold et al., arXiv: 1910.02608.

[14] P. A. R. Ade, M. Aguilar et al. (POLARBEAR Collaboration), Astrophys. J. 848, 121 (2017).

[15] S. Naess, M. Hasselfield, J. McMahon et al., J. Cosmol. Astropart. Phys. 10 (2014) 007.

[16] L. Knox and Y. Song, Phys. Rev. Lett. 89, 011303 (2002).

[17] A. Manzotti, K. T. Story, W. L. K. Wu et al., Astrophys. J. 846, 45 (2017).

[18] N. Aghanim, Y. Akrami et al. (Planck Collaboration), arXiv: 1807.06210.

[19] J. E. Carlstrom, P. A. R. Ade, K. A. Aird et al., Publ. Astron. Soc. Pac. 123, 568 (2011).

[20] S. Padin, Z. Staniszewski, R. Keisler et al., Appl. Opt. 47, 4418 (2008).

[21] J. W. Henning, P. Ade, K. A. Aird et al., Proc. SPIE Int. Soc. Opt. Eng. 8452, 84523A (2012).

[22] J. T. Sayre, P. Ade, K. A. Aird et al., Proc. SPIE Int. Soc. Opt. Eng. 8452, 845239 (2012).

[23] A. T. Crites, J. W. Henning, P. A. R. Ade et al., Astrophys. J. 805, 36 (2015).

[24] K. K. Schaffer, T. M. Crawford, K. A. Aird et al., Astrophys. J. 743, 90 (2011).

[25] K. M. Smith and M. Zaldarriaga, Phys. Rev. D 76, 043001 (2007).

[26] M. Zaldarriaga, Phys. Rev. D 64, 103001 (2001).

[27] E. Hivon, K. M. Górski, C. B. Netterfield, B. P. Crill, S. Prunet, and F. Hansen, Astrophys. J. 567, 2 (2002).
[28] M. Tristram, J. F. Macías-Pérez, C. Renault, and D. Santos, Mon. Not. R. Astron. Soc. 358, 833 (2005).

[29] P. A. R. Ade, N. Aghanim et al. (Planck Collaboration), Astron. Astrophys. 594, A13 (2016).

[30] A. Lewis and S. Bridle, Phys. Rev. D 66, 103511 (2002).

[31] A. Lewis, A. Challinor, and A. Lasenby, Astrophys. J. 538, 473 (2000).

[32] P. A. R. Ade, N. Aghanim et al. (Planck Collaboration), Astron. Astrophys. 571, A22 (2014).

[33] P. A. R. Ade, N. Aghanim et al. (Planck Collaboration), Astron. Astrophys. 571, A16 (2014).

[34] L. M. Mocanu, T. M. Crawford, J. D. Vieira et al., Astrophys. J. 779, 61 (2013).

[35] N. Gupta, C. L. Reichardt, P. A. R. Ade et al., Mon. Not. R. Astron. Soc. 490, 5712 (2019).

[36] A. Kosowsky and A. Loeb, Astrophys. J. 469, 1 (1996).

[37] D. R. Sutton, C. Feng, and C. L. Reichardt, Astrophys. J. 846, 1642017.

[38] P. A. R. Ade, N. Aghanim et al. (Planck Collaboration), Astron. Astrophys. 594, A19 (2016).

[39] A. Zucca, Y. Li, and L. Pogosian, Phys. Rev. D 95, 063506 (2017).

[40] P. A. R. Ade, K. Arnold, M. Atlas et al., Phys. Rev. D 92 , 123509 (2015).

[41] A. N. Bender, P. A. R. Ade, Z. Ahmed et al., Proc. SPIE Int. Soc. Opt. Eng. 10708, 1070803 (2018).

[42] J. D. Hunter, Comput. Sci. Eng. 9, 90 (2007).

[43] E. Jones, T. Oliphant, P. Peterson et al., SciPy: Open source scientific tools for PYTHON (2001), http://www .scipy.org/.

[44] S. van der Walt, S. Colbert, and G. Varoquaux, Comput. Sci. Eng. 13, 22 (2011).

[45] HDF Group, Hierarchical Data Format, version 5, 1997. 